Secondly if attendants accompanying the patient to the hospital appear suspicious, they should be denied entry at the entrance itself.

6. Medical personnel should try to identify signs of violence amongst patients and their relatives. In Australia, a study was conducted among nurses in the emergency ward of a public hospital (9) to identify the signs of observable behaviour indicating a potential for violence in patients and their relatives. Five distinct elements of observable behaviour were identified under the acronym STAMP: $\mathrm{S}$ - staring and eye contact, $\mathrm{T}$ - tone and volume of voice, A - anxiety, M - mumbling, P - pacing (9). On a trial basis, this method can be applied in Indian hospitals with a high prevalence of violence against doctors and, if found beneficial, can be applied to other hospitals as well.

7, Where an attack does break out, a standard protocol should be followed by all staff members in the hospital to prevent any injury to doctors and other personnel, as well as to protect hospital property. This protocol should be practised by hospital staff regularly. One such protocol is Code Purple used by many hospitals to alert the medical staff against potential violence and thus, control the situation.(10)

\section{Statement of funding: None}

\section{References}

1. Bose R. Doctors' anger has long been brewing in Bengal, the NRS fiasco just brought it to boil. News18.com.2019 Jun16 [cited 2019 Sep 3]. Available from: https://www.news18.com/news/buzz/doctors-protestskolkata-assault-hospitals-mamata-banerjee-physical-assault-bengalindian-medical-association-nrs- 2188441.html

2. Ranjan R, Singh M, Pal R, Das JK, Gupta S. Epidemiology of violence against medical practitioners in a developing country (2006-2017). J Health Res Rev. 2018 Sep 1; 5(3):153

3. Kumar M, Verma M, Das T, Pardeshi G, Kishore J, Padmanandan A. A study of workplace violence experienced by doctors and associated risk factors in a tertiary care hospital of South Delhi, India. J Clinical Diagn Res. 2016 Nov; 10(11):LC06-10.

4. Nagral S. Doctors and violence. Indian J Med Ethics. 2001;9(4):107

5. Pai SA.Violence against doctors on the increase in India. Nat Med J India. 2015; 28(4):214.

6. Madhiwalla N, Roy N. Assaults on public hospital staff by patients and their relatives: an inquiry. Indian J Med Ethics. 2006 Apr-Jun;3(2): 51-4

7. Ambesh P.Violence against doctors in the Indian subcontinent: A rising bane. Indian Heart J. 2016 Sep-Oct;68(5):749-50.

8. Ghosh K.Violence against doctors: A wake-up call. Indian J Med Res. 2018 Aug;148(2):130-3.

9. Luck L, Jackson D, Usher K. STAMP: components of observable behaviour that indicate potential for patient violence in emergency departments.J Adv Nurs. 2007 Jul;59(1):11-9.

10. Cates M, Malcolm D, Poirier T, Kendall K. A guarantee to a community. J Emerg Nurs. 2009 Jul 1;35(4):336-8.

\title{
The deteriorating patient-physician relationship in India: Is it time to look within?
}

\section{SHREYAK SHARMA}

\begin{abstract}
The patient-physician relationship in India is in a state of rapid decline with fresh incidents of violence highlighting the scale of the problem. The medical fraternity needs to reflect on certain issues plaguing its conduct with patients and colleagues and embark on steps to address them. In this article, I highlight some major points which could help us arrest the deteriorating trust and heightened violence in hospitals.
\end{abstract}

Keywords: patient-physician relationship, ethics, workplace violence

Healthcare is the backbone of any country. India is one of the biggest producers of healthcare professionals around the world (1). Earlier, the parents of most youngsters, if

Author: Shreyak Sharma (ssharma38@bwh.harvard.edu), Research Fellow, Division of Renal Medicine, Brigham and Women's Hospital, Harvard Medical School, Boston, MA, USA.

To cite: Sharma S. The deteriorating patient - physician relationship in India: Is it time to look within? Indian J Med Ethics. 2019 Oct-Dec;4(4) NS:330-2. DOI: 10.20529/IJME.2019.056

Published online on September 11, 2019.

(C) Indian Journal of Medical Ethics 2019 asked, would wish for their children to become doctors. This established situation is changing now. Our relationship with the people we serve is deteriorating rapidly, so much so that physical and verbal assault has become part and parcel of practising medicine, especially in government hospitals.

While there are numerous external factors to blame, ranging from political apathy to sensationalism in the media, there are serious internal issues within the fraternity that need to be highlighted and reflected upon. We are at a watershed moment as far as practising medicine is concerned, and the recent increase in attacks on doctors has drawn country-wide condemnation.

\section{The age of consumerism is here}

It is a well-known fact that doctors were considered noble souls and revered as "gods" until very recently. However, the world we live in has changed significantly. The $21^{\text {st }}$ century is the age of consumerism where everyone wants to protect their own interests and get the best service for the fees they pay. Greenhalgh and Wessely (2) argued that patients are independent and rational beings who make economic decisions about their health and medical needs to fulfil their own interests. This has created a power shift in the traditional patient-physician relationship and patients no longer see 
their doctors as authoritative omniscient figures who cannot be questioned. In this scenario, our first objective is to accept the new order and not expect traditional ways of practising medicine in India to be sufficient for the present and future generations. Akin to patients wanting to prioritise their economic and health interests, physicians also have the right to gain economically from their profession, but not at the cost of medical ethics. While a large majority of physicians treat their patients ethically, a few examples of unethical treatment practices can be devastating for the fraternity and put the integrity of the profession at large in question. Physicians should take cognisance of this fact and urge the whole community to continue doing good work and refrain from actions that might diminish people's trust in the profession.

\section{Communication and empathy}

Communication skills are a vital part of the patient-physician relationship. Be it the lack of focused training in this respect, or the sheer number of patients that doctors have to deal with, Indian physicians have often been accused of rudeness and indifference to their patients. Any honest Indian physician would agree that they may have reacted in a way which could negatively impact the patient's state of mind, even if there were serious pressures behind it. Empathy and humility are important pillars of the physician-patient relationship and have been scientifically proven to improve the healthcare encounter, patient compliance and outcomes (3). It is high time that the medical curriculum lays a strong emphasis on teaching communication, empathy, humility, and methods to carefully tackle highly charged hospital environments and difficult patients. This sort of training is severely lacking and immediate efforts to integrate it into the curriculum would go a long way towards addressing these issues. Case presentations in medical colleges are mostly limited to summarising the patient's condition in front of teachers, with little emphasis on how the patient interview was conducted. It would be worthwhile to conduct structured patient encounters where the entire patientstudent encounter is monitored and reviewed by faculty.

\section{Need for respectful interaction}

Of the many issues plaguing our fraternity, none is as significant as mutual conduct amongst physicians. Medical colleges are the cradle of future doctors and only a few worthy applicants get an opportunity to fulfill their dreams. However, these youngsters are soon subjected to the harsh realities of medical life, one of which is the concept of seniority. It is this concept of seniority and perceived superiority arising from it that disregards respect for each other and promotes verbal abuse and disdain, right from the first year. Cut to residency, and the same approach is evident, albeit this time in the presence of patients. It is a common sight for patients to see a senior doctor being angry and treating a junior colleague with utter disrespect and sometimes, subjecting them to blatant humiliation. This creates a negative impact on the patients as they start seeing junior doctors as inefficient and in turn treat them in a disrespectful manner.
Residents are in the line of fire daily, are expected to work 16 hours a day and still make time for studying, journal clubs, and thesis. A person dealing with such stress, in addition to the rebukes while on rounds, will not be in a positive frame of mind. A lot of this frustration gets further projected onto patients and our conversations with them, further deteriorating that relationship. A periodic assessment and acknowledgement of challenges faced by them and an attempt at counselling, if indicated, may prove to be the crucial missing tool for residents' health. It will also help to identify doctors suffering from mental health issues like depression and provide timely intervention $(4,5)$. A change in our own attitudes towards each other can be a significant step in the right direction for the medical fraternity.

\section{Time to focus on emergency medicine?}

It has been well established that most cases of healthcare violence occur in the emergency department $(6,7)$. These adrenaline-packed environments are often left to medical officers and interns to handle. The lack of proper triage and ability to handle patients and their relatives leads to verbal and physical conflict. The Medical Council of India accepted the proposal to introduce Emergency Medicine (EM) as a specialty in 2009 (8); but until now, only a handful of states have implemented it. Introducing dedicated and trained EM residents who are sensitised and taught to handle tough situations, charged relatives, and "breaking bad news" is the need of the hour to enable better handling of emergency rooms and trauma centers.

This is an important time for the healthcare community in India and it is vital that drastic measures be taken by the government, professional bodies, civil society, and the media to preserve trust in the system and resolve all outstanding issues related to healthcare. While we can only hope that the Government improves health infrastructure and investment, and the media refrains from its TRP-driven attempts to sensationalise, such expectations are at best idealistic. It is up to us to introspect and make changes that might help in promoting a safer and more efficient work environment.

\section{References}

1. Central Bureau of Health Intelligence, Ministry of Health and Family Welfare. National Health Profile. New Delho: CBHl; 2018. p. 217-28.

2. Greenhalgh T, Wessely S. 'Health for me': a sociocultural analysis of healthism in the middle classes. BrMed Bull. 2004.69:197-213.

3. Ruberton PM, Huynh HP, Miller TA, Kruse E, Chancellor J, Lyubomirsky $S$ The relationship between physician humility, physician-patient communication, and patient health. Patient Educ Couns. 2016 Jul; 99(7):1138-45

4. Sharp M, Burkart KM. Trainee wellness: why it matters, and how to promote it. Ann Am Thorac Soc. 2017 Apr;14(4):505-12.

5. Daskivich TJ, Jardine DA, Tseng J, Correa R, Stagg BC, Jacob KM, Harwood $J$. Promotion of wellness and mental health awareness among physicians in training: perspective of a national, multispecialty panel of residents and fellows. J Grad Med Educ. 2015 Mar;7(1):143-7.

6. Phillips JP.Workplace violence against health care workers in the United States. N Engl J Med. 2016 Apr 26;374(17):1661-9.

7. Chauhan V, Galwankar S, Kumar R, Raina SK, Aggarwal P, Agrawal N Krishnan SV, Bhoi S, Kalra OP, Soans ST, Aggarwal V, Kubendra M, Bijayraj R, Datta S, Srivastava RP. The 2017 Academic College of Emergency 
Experts and Academy of Family Physicians of India position statement on preventing violence against health-care workers and vandalization of health-care facilities in India. Int J Crit IIIn Inj Sci. 2017 Apr-Jun; 7(2):7983.
8. Sriram V, Hyder AA, Bennett S. The Making of a New Medical Specialty: A Policy Analysis of the Development of Emergency Medicine in India. Int J Health Policy Manag. 2018 Nov; 7(11):993-1006.

\section{On the absence of a doctor's dilemma in India: Reflections on impatient violence}

\section{R Srivatsan}

On June 10, 2019, Mohammed Sayeed, a 75-year-old patient was admitted to the Nil Ratan Sircar Medical College and Hospital, Kolkata, West Bengal (1). He died that night due to a cardiac arrest and this led to a scuffle between the patient's family and duty doctors. In retaliation, the doctors refused to discharge the body, asserting that since the family claimed it was a suspicious death, a post-mortem was required. A mob arrived, and in the confrontation, a doctor was injured. The medicos struck work. Doctors and medical associations across the country have voiced unanimous support for the doctors, and called for protective legislation against the violence of the public. As a consequence of these nationwide doctors' protests, the Supreme Court has now proposed a law that protects doctors by severely punishing those who attack them (2). Meanwhile, it does seem as if such attacks are increasing.

The Telegraph carried three accounts of the incident: that of the duty doctors, the relatives, and the police (3). The differences in perspective are striking, as are the "facts" of the case. The doctors' version is indignant, the patients' family say their heads were "overheated" (matha garam ho gaya), and the police say there was a pitched battle when the doctors attacked the mob and police (3). Which is the true story? As the English historian EH Carr once said, facts are fish that swim around in the ocean (4), especially in such a rapidly politicising situation. The fish you catch determine the story you tell.

The wise doctor's dilemma should be to decide on the correct story to tell and the appropriate step to take. But for some reason, there is only the thoughtless conviction that they have been wronged, are perpetually at risk, and that draconian protection is needed.

\section{Statistical insight}

In order to put the patient's death, the doctors' demands and the government's response in perspective, it would be

Author: Srivatsan R (r.srivats@gmail.com), Independent Researcher on the politics of healthcare, development administration and tribal welfare in India; former Senior Fellow, Anveshi Research Centre for Women's Studies, Hyderabad INDIA.

To cite: Srivatsan R. On the absence of a doctor's dilemma in India: Reflections on impatient violence. Indian J Med Ethics. 2019 Oct-Dec;4(4) NS:332-3. DOI: 10.20529/JJME.2019.066

OIndian Journal of Medical Ethics 2019 useful to look at some statistical data. The Indian census, civil registration and sampling exercises (5), despite imperfections, are useful; more especially so the Sample Registration System statistical report (6), which, using large sample sizes, estimates vital statistics across the country. There have been criticisms and queries about the accuracy of these data collection systems (7), but for our purposes they will suffice even if they are only fifty percent accurate. The last section of the SRS Report of 2016 shows the distribution of deaths between public and private hospitals, external medical supervision, unqualified supervision and other situations (6:p 180).

Let us look at the West Bengal statistics:

- Population: 91.28 million in the 2011 Census (likely to be higher today).

- Estimated Crude Death Rate (6: p 122): 5.8 per 1000 population or about 529,400 as per the 2011 population. Reporting of deaths within the country is reasonably accurate with the CRS indicating that 82 percent of deaths were registered in 2016. ${ }^{1}$ (8: Statement $2, \mathrm{p} 2$ ).

- Nearly $33 \%$ of these deaths occur in government hospitals (6: p 180)-approximately 176,467 . With even a $50 \%$ error in the sample-based estimates ("inefficient government systems", eg), this figure is an adequate indicator of the scale of the problem of violence. This of course includes hospital-based deaths of infant and mothers, the aged, those afflicted with terminal diseases, and due to acute events. Break-ups along these categories are not easily available but could be one additional pointer to a more nuanced causation.

Now remember that most of these 176,467 hospital-based deaths were mourned by family, friends and community. So, even if say $200+$ violent confrontations have occurred the past year before the current incident ${ }^{2}, 176,267$ deaths have occurred in hospitals without violence. What then is the reason for the striking junior doctors' explosive rage in these specific instances? The only way I understand it is that many patient deaths in hospitals are handled by a relatively small number of frontline duty doctors. Exceptional violence in this event is faced by an even smaller number. As all these duty doctors experience and manage the routine flow of patient deaths, violent encounters stand out as an assault on their identity as committed professionals. 